\title{
Carlsson's rank conjecture and a conjecture on square-zero upper triangular matrices
}

\author{
Berrin Şentürk *, Özgün Ünlü ${ }^{1}$ \\ Department of Mathematics, Bilkent University, Ankara, 06800, Turkey
}

\section{A R T I C L E I N F O}

\section{Article history:}

Received 26 May 2017

Received in revised form 20 July

2018

Available online 13 September 2018

Communicated by S. Iyengar

\section{$M S C$ :}

55M35; 13D22; 13D02

\section{Keywords:}

Rank conjecture

Square-zero matrices

Projective variety

Borel orbit

\begin{abstract}
A B S T R A C T
Let $k$ be an algebraically closed field and $A$ the polynomial algebra in $r$ variables with coefficients in $k$. In case the characteristic of $k$ is 2, Carlsson [9] conjectured that for any $D G$ - $A$-module $M$ of dimension $N$ as a free $A$-module, if the homology of $M$ is nontrivial and finite dimensional as a $k$-vector space, then $2^{r} \leq N$. Here we state a stronger conjecture about varieties of square-zero upper triangular $N \times N$ matrices with entries in $A$. Using stratifications of these varieties via Borel orbits, we show that the stronger conjecture holds when $N<8$ or $r<3$ without any restriction on the characteristic of $k$. As a consequence, we obtain a new proof for many of the known cases of Carlsson's conjecture and give new results when $N>4$ and $r=2$.
\end{abstract}

() 2018 Elsevier B.V. All rights reserved.

\section{Introduction}

A well-known conjecture in algebraic topology states that if $(\mathbb{Z} / p \mathbb{Z})^{r}$ acts freely and cellularly on a finite $\mathrm{CW}$-complex homotopy equivalent to $S^{n_{1}} \times \ldots \times S^{n_{m}}$, then $r$ is less than or equal to $m$. This conjecture is known to be true in several cases: In the equidimensional case $n_{1}=\ldots=n_{m}=: n$, Carlsson [7], Browder [6], and Benson-Carlson [5] proved the conjecture under the assumption that the induced action on homology is trivial. Without the homology assumption, the equidimensional conjecture was proved by Conner [11] for $m=2$, Adem-Browder [1] for $p \neq 2$ or $n \neq 1,3,7$, and Yalçın [25] for $p=2, n=1$. In the non-equidimensional case, the conjecture is proved by Smith [23] for $m=1$, Heller [13] for $m=2$, Carlsson [10] for $p=2$ and $m=3$, Refai [20] for $p=2$ and $m=4$, and Okutan-Yalçın [19] for products in which the average of the dimensions is sufficiently large compared to the differences between them. The general case $m \geq 5$ is still open.

\footnotetext{
* Corresponding author.

E-mail addresses: berrin@fen.bilkent.edu.tr (B. Şentürk), unluo@fen.bilkent.edu.tr (Ö. Ünlü).

1 The second author is partially supported by TÜBA-GEBİP/2013-22.
} 
Let $G=(\mathbb{Z} / p \mathbb{Z})^{r}$ and $k$ be an algebraically closed field of characteristic $p$. Assume that $G$ acts freely and cellularly on a finite $\mathrm{CW}$-complex $X$ homotopy equivalent to a product of $m$ spheres. One can consider the cellular chain complex $C_{*}(X ; k)$ as a finite chain complex of free $k G$-modules with homology $H_{*}(X ; k)$ that has dimension $2^{m}$ as a $k$-vector space. Hence, a stronger and purely algebraic conjecture can be stated as follows: If $C_{*}$ is a finite chain complex of free $k G$-modules with nonzero homology then $\operatorname{dim}_{k} H_{*}\left(C_{*}\right) \geq 2^{r}$. However, Iyengar-Walker in [15] disproved this algebraic conjecture when $p \neq 2$, but the algebraic version for $p=2$ remains open.

Let $R$ be a graded ring. A pair $(M, \partial)$ is a differential graded $R$-module if $M$ is a graded $R$-module and $\partial$ is an $R$-linear endomorphism of $M$ that has degree -1 and satisfies $\partial^{2}=0$. Moreover, a $D G$ - $R$-module is free if the underlying $R$-module is free.

Let $A=k\left[y_{1}, \ldots, y_{r}\right]$ be the polynomial algebra in $r$ variables, where $k$ is a field and each $y_{i}$ has degree -1 . Using a functor from the category of chain complexes of $k G$-modules to the category of differential graded $A$-modules, Carlsson showed in [8], [9] that the above algebraic conjecture is equivalent to the following conjecture when the characteristic of $k$ is 2 :

Conjecture 1. Let $k$ be an algebraically closed field, A the polynomial algebra in $r$ variables with coefficients in $k$, and $N$ a positive integer. If $(M, \partial)$ is a free $D G$-A-module of rank $N$ whose homology is nonzero and finite dimensional as a $k$-vector space, then $N \geq 2^{r}$.

When the characteristic of $k$ is 2, Conjecture 1 was proved by Carlsson [10] for $r \leq 3$ and Refai [20] for $N \leq 8$. Avramov, Buchweitz, and Iyengar in [4] dealt with regular rings and in particular they proved Conjecture 1 for $r \leq 3$ without any restriction on the characteristic of $k$. See also Proposition 1.1 and Corollary 1.2 in [2], Theorem 5.3 in [24] for results in characteristic not equal to 2.

In this paper we consider the conjecture from the viewpoint of algebraic geometry. We show that Conjecture 1 is implied by the following in Section 2:

Conjecture 2. Let $k$ be an algebraically closed field, $r$ a positive integer, and $N=2 n$ an even positive integer. Assume that there exists a nonconstant morphism $\psi$ from the projective variety $\mathbb{P}_{k}^{r-1}$ to the weighted quasi-projective variety of rank $n$ square-zero upper triangular $N \times N$ matrices $\left(x_{i j}\right)$ with $\operatorname{deg}\left(x_{i j}\right)=d_{i}-d_{j}+1$ for some $N$-tuple of nonincreasing integers $\left(d_{1}, d_{2}, \ldots, d_{N}\right)$. Then $N \geq 2^{r}$.

We will give a more precise statement of Conjecture 2 in Section 3 after discussing necessary definitions and notation. We propose the following, which is stronger than Conjecture 2:

Conjecture 3. Let $k, r, N, n$ and $\psi$ be as in Conjecture 2. Assume $1 \leq \mathcal{R}, \mathcal{C} \leq N$ and the value of $x_{i j}$ at every point in the image of $\psi$ is 0 whenever $i \geq N-\mathcal{R}+1$ or $j \leq \mathcal{C}$. Then $N \geq 2^{r-1}(\mathcal{R}+\mathcal{C})$.

Note that in Conjecture 3 we have $2^{r-1}(\mathcal{R}+\mathcal{C}) \geq 2^{r}$ because $1 \leq \mathcal{R}$ and $1 \leq \mathcal{C}$. The main result of the paper is a proof of Conjecture 3 when $N<8$ or $r<3$, see Theorem 2 and Theorem 6 . As Conjecture 3 is the strongest conjecture mentioned above, we obtain proofs of all the conjectures in this introduction under the same conditions, including the main result of Carlsson in [9]. Also note that for $r=2$, taking $N>4$ gives novel results not covered in the literature. However, when the characteristic of the field $k$ is not 2, Iyengar-Walker [15] gave a counterexample to Conjecture 1 for each $r \geq 8$. Hence by Section 2, we can say that Conjectures 2 and 3 are also false when $r \geq 8$ and the characteristic of the field $k$ is not 2 . All these conjectures are still open in case the characteristic of $k$ is 2 . In Section 4, we conclude with examples and problems.

We thank the referee for giving us extensive feedback, a shorter proof of Theorem 1 and encouraging us to extend our results to fields with characteristics other than 2. We are also grateful to Matthew Gelvin for comments and suggestions. 


\section{Some notes on Conjectures 1, 2, and 3}

To show that Conjecture 3 is the strongest conjecture in Section 1, it is enough to prove the following theorem.

Theorem 1. Conjecture 2 implies Conjecture 1.

Proof. Let $k, r$, and $A$ be as in Conjecture 1. Let $(M, \partial)$ be a free $D G$ - $A$-module of rank $N$ which satisfies the hypothesis in Conjecture 1. Without loss of generality, we can assume that $N$ is the smallest rank of all such $D G$ - $A$-modules.

Suppose the image of the differential $\partial$ is not contained in $\left(y_{1}, \ldots, y_{r}\right) M$. Then, there exists a basis $b_{1}, \ldots, b_{N}$ of $M$ and there are some $i$ and $j$ so that $\partial\left(b_{i}\right)=c b_{j}+\sum_{l \neq j} g_{l} b_{l}$ for some non-zero $c \in k$ and some $g_{l} \in A$. Replacing $b_{j}$ with $\partial\left(b_{i}\right)$ gives a new basis $b_{1}^{\prime}, \ldots, b_{N}^{\prime}$ such that $\partial\left(b_{i}^{\prime}\right)=b_{j}^{\prime}$. Now form the acyclic sub- $D G$ - $A$-module $(E, \partial)$ of $(M, \partial)$ spanned by $b_{i}^{\prime}, b_{j}^{\prime}$. The map $(M, \partial) \rightarrow(M, \partial) /(E, \partial)$ is a surjective quasi-isomorphism and $M / E$ is free of rank $N-2$. This is a contradiction. Hence, the image of the differential $\partial$ is contained in $\left(y_{1}, \ldots, y_{r}\right) M$.

Now pick any basis $c_{1}, \ldots, c_{N}$ of $M$ such that $\operatorname{deg}\left(c_{1}\right) \leq \cdots \leq \operatorname{deg}\left(c_{N}\right)$. Let $m$ be such that $\operatorname{deg}\left(c_{N-m+1}\right)=\cdots=\operatorname{deg}\left(c_{N}\right)$ and $\operatorname{deg}\left(c_{i}\right)<\operatorname{deg}\left(c_{N-m+1}\right)$ for all $i<N-m+1$. For each $i$, we have $\partial\left(c_{i}\right)=\sum_{j} g_{i j} c_{j}$, for some homogeneous polynomials $g_{i j} \in A$. Since the image of $\partial$ is contained in $\left(y_{1}, \ldots, y_{r}\right) M$, no $g_{i j}$ is a non-zero constant. Thus, whenever $g_{i j}$ is non-zero, we have $\operatorname{deg}\left(g_{i j}\right) \leq-1$ and hence $\operatorname{deg}\left(c_{j}\right)=\operatorname{deg}\left(c_{i}\right)-1-\operatorname{deg}\left(g_{i j}\right) \geq \operatorname{deg}\left(c_{i}\right)$. It follows that the differential on $M$ restricts to one on the submodule

$$
L=A c_{N-m+1} \oplus \cdots \oplus A c_{N}
$$

More precisely, for all $i \in\{N-m+1, \ldots, N\}$ we have $\partial\left(c_{i}\right)=\sum_{j=N-m+1}^{N} g_{i j} c_{j}$ where each nonzero $g_{i j}$ is a linear polynomial. Hence, relative to the basis $c_{N-m+1}, \ldots, c_{N}$, the differential $\partial$ on $L$ is given by a matrix in the form $y_{1} X_{1}+\cdots+y_{r} X_{r}$ where each $X_{i}$ is an $m \times m$ matrix with entries in $k$. Since $\partial^{2}=0$ we have $X_{i}^{2}=0$ and $X_{i} X_{j}+X_{j} X_{i}=0$ for all $i, j$. In case the characteristic of the field $k$ is 2 , by a classical result about commuting set of matrices, for example see Theorem 7 on page 207 in [14], there exists an invertible $m \times m$ matrix $T$ with coefficients in $k$ such that $T^{-1} X_{i} T$ is upper triangular for all $i \in\{1, \ldots, r\}$. In case the characteristic of the field $k$ is not 2, for every polynomial $Q$ in noncommutative $r$ variables, the square of the matrix $Q\left(X_{1}, \ldots X_{r}\right)\left(X_{i} X_{j}-X_{j} X_{i}\right)$ is zero. Therefore, by a theorem of McCoy as stated in [22] (see also [12], [17]), again there exists a matrix $T$ as above which simultaneously conjugates all $X_{i}$ 's to upper triangular matrices. In other words, there is a $k$-linear change of basis in which each $X_{i}$ is upper triangular. It follows that, relative to this new basis $c_{N-m+1}^{\prime}, \ldots, c_{N}^{\prime}$ of $L$ one has $\partial\left(c_{i}^{\prime}\right) \in \oplus_{j=i+1}^{N} A c_{j}^{\prime}$ for all $i \in\{N-m+1, \ldots N\}$. Note that $M / L$ is a free $D G$ - $A$-module whose differential has an image in $\left(y_{1}, \ldots, y_{r}\right)(M / L)$ and so, by inductive arguments on rank, we may assume that $M / L$ admits a basis which makes its differential upper triangular. The union of any lift of this basis to $M$ with the basis $c_{N-m+1}^{\prime}, \ldots, c_{N}^{\prime}$ gives a basis $B$ for $M$ where $\partial$ is represented by an upper triangular matrix $\Psi^{\prime}$. Moreover, Propositions 8 and 9 in [10] work for any characteristic. Hence $N$ is divisible by 2 and for any $\gamma$ in $k^{r}-\{0\}$ the evaluation of $\Psi^{\prime}$ at $\gamma$ gives a matrix of rank $N / 2$.

Let $S=k\left[x_{1}, \ldots, x_{r}\right]$ be the polynomial algebra with $\operatorname{deg}\left(x_{i}\right)=1$. For $1 \leq i \leq r$, replace $y_{i}$ with $x_{i}$ in $\Psi^{\prime}$ to obtain $\Psi$. Note that $\Psi$ can be considered as a nonconstant morphism from the projective variety $\mathbb{P}_{k}^{r-1}$ to the weighted quasi-projective variety of rank $N / 2$ square-zero upper triangular $N \times N$ matrices $\left(x_{i j}\right)$ with $\operatorname{deg}\left(x_{i j}\right)=d_{i}-d_{j}+1$ where $d_{i}=-($ degree of theith element in $B)$. 


\section{Varieties of square-zero matrices}

We assume that $k$ is an algebraically closed field, $n$ a positive integer, $N=2 n$, and $d=\left(d_{1}, d_{2}, \ldots, d_{N}\right)$ an $N$-tuple of nonincreasing integers.

\subsection{Statements of conjectures}

We give here the notation for the affine and projective varieties used to prove the conjectures discussed above. First we fix an affine variety $U_{N}$, a ring $R\left(U_{N}\right)$, and a subvariety $V_{N}$ as follows:

- $U_{N}$ is the affine variety of strictly upper triangular $N \times N$ matrices over $k$.

- $R\left(U_{N}\right)=k\left[x_{i j} \mid 1 \leq i<j \leq N\right]$ is the coordinate ring of $U_{N}$.

- $V_{N}$ is the subvariety of square zero matrices in $U_{N}$.

Define an action of the unit group $k^{*}$ on $U_{N}$ by $\lambda \cdot\left(x_{i j}\right)=\left(\lambda^{d_{i}-d_{j}+1} x_{i j}\right)$ for $\lambda \in k^{*}$. Using this action we set two more notation.

- $U(d)$ is the weighted projective space given by the quotient of $U_{N}-\{0\}$ by the action of $k^{*}$.

- $R(U(d))$ is the homogeneous coordinate ring of $U(d)$. In other words, $R(U(d))$ is $R\left(U_{N}\right)$ considered as a graded ring with $\operatorname{deg}\left(x_{i j}\right)=d_{i}-d_{j}+1$.

Note that the polynomial $p_{i j}=\sum_{m=i+1}^{j-1} x_{i m} x_{m j}$ in $R(U(d))$ is homogeneous of degree $d_{i}-d_{j}+2$ whenever $1 \leq i<j \leq N$. Similarly, the $n \times n$-minors of $\left(x_{i j}\right)$ are homogeneous polynomials in $R(U(d))$. Hence, we define two subvarieties of $U(d)$ as follows:

- $V(d)$ is the projective variety of square zero matrices in $U(d)$.

- $L(d)$ is the subvariety of matrices of rank less than $n$ in $V(d)$.

We can use this terminology to restate Conjecture 2:

Conjecture 4. Let $k$ be an algebraically closed field, $r$ a positive integer, and $d$ an $N$-tuple of nonincreasing integers. If there exists a nonconstant morphism $\psi$ from the projective variety $\mathbb{P}_{k}^{r-1}$ to the quasi-projective variety $V(d)-L(d)$, then $N \geq 2^{r}$.

Let $U$ be an open subset of $V(d)$. We say $\psi: \mathbb{P}_{k}^{r-1} \rightarrow U$ is a nonconstant morphism if $\psi$ is represented by $\left(\psi_{i j}\right)$ so that the following conditions are satisfied:

(I) there exists a positive integer $m$ so that each $\psi_{i j}$ is a homogeneous polynomial in the variables $x_{1}, x_{2}, \ldots, x_{r}$ in $S$ of degree $m\left(d_{i}-d_{j}+1\right)$ for $1 \leq i<j \leq N$,

(II) for every $\gamma \in \mathbb{P}_{k}^{r-1}$ there exists $i, j$ such that $\psi_{i j}(\gamma) \neq 0$.

In particular, if $\psi: \mathbb{P}_{k}^{r-1} \rightarrow U$ is a nonconstant morphism, $\psi$ can be considered as a function from $\mathbb{P}_{k}^{r-1}$ to $U$ represented by a nonconstant polynomial map $\widetilde{\psi}$ from $\mathbb{A}_{k}^{r}$ to the cone over $U$ such that $\widetilde{\psi}\left(\mathbb{A}_{k}^{r}-\{0\}\right)$ does not contain the zero matrix in $V_{N}$. Each indeterminate $x_{i j}$ can be viewed as homogeneous polynomial in $R(U(d))$. Hence for $1 \leq \mathcal{R}, \mathcal{C} \leq N$ we define an important subvariety of $V(d)$ :

- $V(d)_{\mathcal{R C}}$ is the subvariety of $V(d)$ given by the equations $x_{i j}=0$ for $i \geq N-\mathcal{R}+1$ or $j \leq \mathcal{C}$. 
Now we restate the Conjecture 3 as follows:

Conjecture 5. Let $k$ be an algebraically closed field, $r$ a positive integer, and $d$ an $N$-tuple of nonincreasing integers. If there exists a nonconstant morphism $\psi$ from the projective variety $\mathbb{P}_{k}^{r-1}$ to the quasi-projective variety $V(d)_{\mathcal{R C}}-L(d)$, then $N \geq 2^{r-1}(\mathcal{R}+\mathcal{C})$.

Hence, these varieties are the main interest in this paper.

\subsection{Action of a Borel subgroup on $V_{N}$}

Here we introduce an action of a Borel subgroup in the group of invertible $N \times N$ matrices on the varieties discussed in the previous subsection. First we set a notation for the Borel subgroup.

- $B_{N}$ is the group of invertible upper triangular $N \times N$ matrices with coefficients in $k$.

The group $B_{N}$ acts on $V_{N}$ by conjugation.

- $V_{N} / B_{N}$ denotes the set of orbits of the action of $B_{N}$ on $V_{N}$.

- $B_{X}$ denotes the $B_{N}$-orbit that contains $X \in V_{N}$.

A partial permutation matrix is a matrix having at most one nonzero entry, which is 1 , in each row and column. A result of Rothbach (Theorem 1 in [21]) implies that each $B_{N}$-orbit of $V_{N}$ contains a unique partial permutation matrix. Hence we introduce the following notation:

- $\mathbf{P M}(N)$ denotes the set of nonzero $N \times N$ strictly upper triangular square-zero partial permutation matrices.

There is a one-to-one correspondence between $\mathbf{P M}(N)$ and $V_{N} / B_{N}$ sending $P$ to $B_{P}$. We can identify these partial permutation matrices with a subset of the symmetric group $\operatorname{Sym}(N)$ :

- $\mathbf{P}(N)$ is the set of involutions in $\operatorname{Sym}(N)$; i.e., the set of non-identity permutations whose square is the identity ().

For $P \in \mathbf{P M}(N)$ and $\sigma \in \mathbf{P}(N)$,

- $\sigma_{P}$ denotes the permutation in $\mathbf{P}(N)$ that sends $i$ to $j$ if $P_{i j}=1$;

- $P_{\sigma}$ denotes the partial permutation matrix in $\mathbf{P M}(N)$ that satisfies $\left(P_{\sigma}\right)_{i j}=1$ if and only if $\sigma(i)=j$ and $i<j$.

Clearly the assignments $P \mapsto \sigma_{P}$ and $\sigma \mapsto P_{\sigma}$ are mutual inverses and so define a one-to-one correspondence between $\mathbf{P}(N)$ and $\mathbf{P} \mathbf{M}(N)$.

\subsection{A partial order on the set of orbits}

There are important partial orders on $V_{N} / B_{N}, \mathbf{P}(N), \mathbf{P M}(N)$, all of which are equivalent under the one-to-one correspondence mentioned above (cf. [21]). We begin with $V_{N} / B_{N}$. For Borel orbits $B, B^{\prime} \in$ $V_{N} / B_{N}$, 
- $B^{\prime} \leq B$ means the closure of $B$, considered as a subspace of $V_{N}$, contains $B^{\prime}$.

Second, we define a partial order on $\mathbf{P M}(N)$. To do this, we consider ranks of certain minors of partial permutation matrices. In general, for an $N \times N$ matrix $X$,

- $r_{i j}(X)$ denotes the rank of the lower left $((N-i+1) \times j)$ submatrix of $X$, where $1 \leq i<j \leq N$.

For partial permutation matrices $P^{\prime}, P \in \mathbf{P M}(N)$,

- $P^{\prime} \leq P$ means $r_{i j}\left(P^{\prime}\right) \leq r_{i j}(P)$ for all $i, j$.

Third, we define a partial order on $\mathbf{P}(N)$. For positive integers $i<j$, let $\sigma(i, j)$ denote the product of the permutations $\sigma$ and $(i, j)$ and $\sigma^{(i, j)}$ the conjugate of $\sigma$ by $(i, j)$. For $\sigma, \sigma^{\prime} \in \mathbf{P}(N)$,

- $\sigma^{\prime} \leq \sigma$ if $\sigma^{\prime}$ can be obtained from $\sigma$ by a sequence of moves of the following form:

- Type I replaces $\sigma$ with $\sigma(i, j)$ if $\sigma(i)=j$ and $i \neq j$.

- Type II replaces $\sigma$ with $\sigma^{\left(i, i^{\prime}\right)}$ if $\sigma(i)=i<i^{\prime}<\sigma\left(i^{\prime}\right)$.

- Type III replaces $\sigma$ with $\sigma^{\left(j, j^{\prime}\right)}$ if $\sigma(j)<\sigma\left(j^{\prime}\right)<j^{\prime}<j$.

- Type IV replaces $\sigma$ with $\sigma^{\left(j, j^{\prime}\right)}$ if $\sigma\left(j^{\prime}\right)<j^{\prime}<j=\sigma(j)$.

- Type V replaces $\sigma$ with $\sigma^{(i, j)}$ if $i<\sigma(i)<\sigma(j)<j$.

The idea of describing order via these moves comes from [16]. Although we use different names for moves, the set of possible moves are same. We represent a permutation $\left(i_{1}, j_{1}\right)\left(i_{2}, j_{2}\right) \ldots\left(i_{s}, j_{s}\right)$ in $\mathbf{P}(N)$ by the matrix

$$
\left(\begin{array}{cccc}
i_{1} & i_{2} & \ldots & i_{s} \\
j_{1} & j_{2} & \ldots & j_{s}
\end{array}\right) .
$$

For example, we draw the Hasse diagram of $\mathbf{P}(4)$ in which each edge is labelled by the type of the move it represents (see Fig. 1).

When $N \geq 6$, the Hasse diagram for $\mathbf{P}(N)$ is too large to draw here. We are actually only interested in a small part of this diagram, which we discuss in Section 3.6.

One can consider Fig. 1 as a stratification of $V_{4}$. In the next section, we use the stratification of $V_{N}$ to stratify $V(d)$.

\subsection{Stratification of $V(d)$}

For $d=\left(d_{1}, d_{2}, \ldots, d_{N}\right)$ an $N$-tuple of nonincreasing integers, $\lambda \in k^{*}$, and $X=\left(x_{i j}\right) \in V_{N}$, we have

$$
\lambda \cdot X=\lambda \cdot\left(x_{i j}\right)=\left(\lambda^{d_{i}-d_{j}+1} x_{i j}\right)=D_{\lambda} I_{\lambda} X D_{\lambda}^{-1}
$$

where $D_{\lambda}$ denotes the diagonal matrix with entries $\lambda^{d_{1}}, \lambda^{d_{2}}, \ldots, \lambda^{d_{N}}$ and $I_{\lambda}$ is the scalar matrix with all diagonal entries $\lambda$. Let $P_{X} \in \mathbf{P M}(N)$ be the unique partial permutation matrix in the Borel orbit of $X$. Consider $b \in B_{N}$ such that

$$
P_{X}=b^{-1} X b
$$

Let $I_{\lambda, X}$ be the diagonal matrix whose $j$ th entry is $\lambda$ if $\left(P_{X}\right)_{i j}=1$ for some $i$ and 1 otherwise. Then we have 


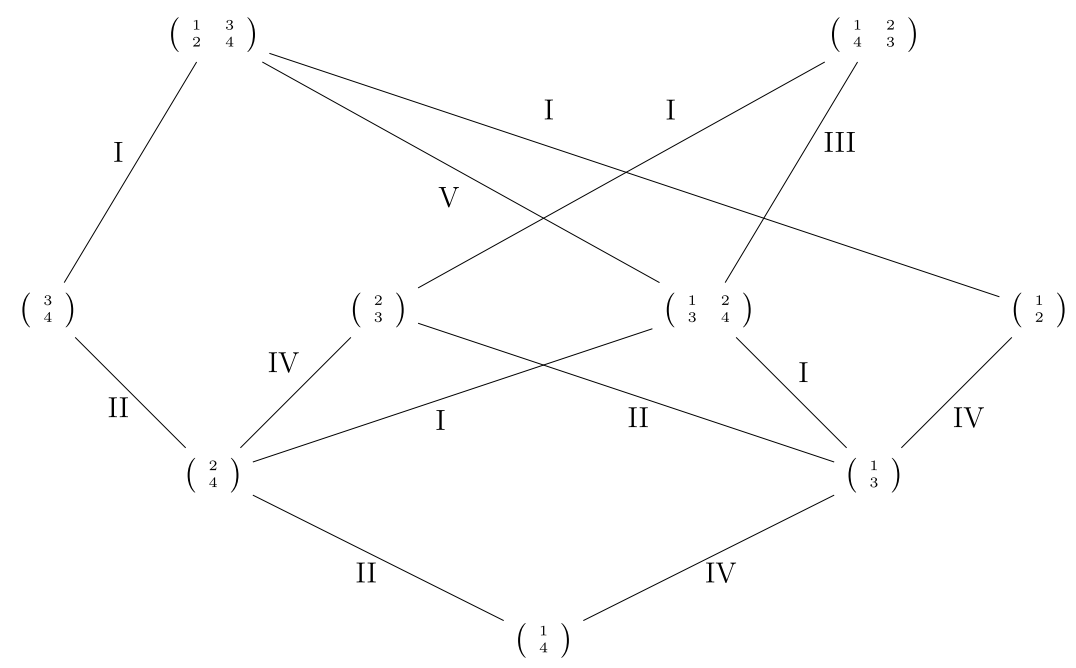

Fig. 1. Hasse diagram of $\mathbf{P}(4)$

$$
I_{\lambda} P_{X}=I_{\lambda, X}^{-1} P_{X} I_{\lambda, X}
$$

Hence, we have

$$
\lambda \cdot X=D_{\lambda} b I_{\lambda, X}^{-1} b^{-1} X b I_{\lambda, X} b^{-1} D_{\lambda}^{-1}=Z^{-1} X Z
$$

where $Z=b I_{\lambda, X} b^{-1} D_{\lambda}^{-1}$ is in $B_{N}$. Thus, for any $X \in V(d)$ there exists a well-defined Borel orbit in $V_{N} / B_{N}$ that contains a representative of $X$ in $V_{N}$. Hence we can set the following notation. For $X \in V(d)$,

- $B_{X}$ denotes the Borel orbit in $V_{N} / B_{N}$ that contains a representative of $X$ in $V_{N}$.

Let $\psi: \mathbb{P}_{k}^{r-1} \rightarrow V(d)-L(d)$ be a nonconstant morphism. There is a lift of this morphism to a morphism from $\mathbb{A}_{k}^{r}-\{0\}$ to the cone over $V(d)-L(d)$ that can be extended to a morphism $\widetilde{\psi}: \mathbb{A}_{k}^{r} \rightarrow V_{N}$. Since $\mathbb{A}_{k}^{r}$ is an irreducible affine variety, there exists a unique maximal Borel orbit among the Borel orbits that intersects the image of $\widetilde{\psi}$ nontrivially. Note that this maximal Borel orbit is independent of the lift and extension we selected because it is also maximal in the set $\left\{B_{X} \mid X \in V(d)\right\}$. Hence we may associate a permutation to the nonconstant morphism $\psi$ :

- $\sigma_{\psi}$ is the permutation that corresponds to the unique maximal Borel orbit $B_{X}$ where $X$ is in the image of $\psi$.

Note that every point in the image of a morphism $\psi$ as above must have rank $n$. Hence $\sigma_{\psi}$ must be a product of $n$ distinct transpositions. In Section 3.6, we will restrict our attention to such permutations.

\subsection{Operations on polynomial maps from $\mathbb{A}_{k}^{r}$ to $V_{N}$}

Another way to see that $B_{X}$ is well-defined for $X \in V(d)$ is to consider the fact that a minor of a representative of $X$ is zero if and only if the corresponding minor of another representative is zero. We use this fact several times to prove our main result. Hence we introduce the following notation. For $X \in V_{N}$,

- $m_{j_{1} j_{2} \ldots j_{k}}^{i_{1} i_{2} \ldots i_{k}}(X)$ denotes the determinant of the $k \times k$ submatrix obtained by taking the $i_{1}$ th, $i_{2}$ th, $\ldots, i_{k}$ th rows and $j_{1}$ th, $j_{2}$ th $, \ldots, j_{k}$ th columns of $X$. 


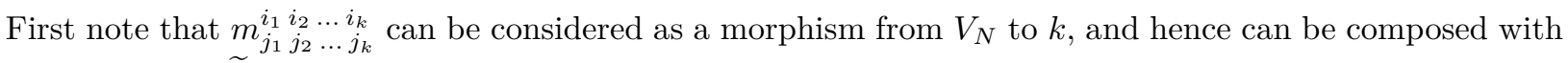
the morphism $\widetilde{\psi}$ mentioned in the previous subsection. Here we discuss several other morphisms that we can compose with such morphisms. For $u \in k$,

- $R_{i, j}(u)$ is the function that takes a square matrix $M$ and multiplies the $i$ th row of $M$ by $u$ and adds it to the $j$ th row of $M$ while multiplying the $j$ th column of $M$ by $u$ and adding it to the $i$ th column of $M$.

Note that $R_{i, j}(u)(M)$ is a conjugate of $M$. In fact, they are in the same Borel orbit when $M \in V_{N}$ and $i>j$. Hence, for $i>j$, we can consider $R_{i, j}(u)$ as an operation that takes a morphism from $\mathbb{A}_{k}^{s}$ to $V_{N}$ and transforms it to a morphism from $\mathbb{A}_{k}^{s+1}$ to $V_{N}$ by considering $u$ as a new indeterminate and applying $R_{i, j}(u)$ to the morphism. For $v \in k^{*}$,

- $D_{i}(v)$ denotes the function that takes a square matrix $M$ and multiplies the $i$ th row of $M$ by $v$ and the $i$ th column of $M$ by $1 / v$.

Let $q$ be a polynomial in $s$ indeterminates. We define $D_{i}(q)$ as an operation that takes a rational map from the quasi-affine variety $\mathbb{A}_{k}^{s}-Z$ to $V_{N}$ and transforms it into a rational map from $\mathbb{A}_{k}^{s}-Z \cup V(q)$ to $V_{N}$ by applying $D_{i}(q)$, using the following notation:

- $V\left(q_{1}, q_{2}, \ldots, q_{k}\right)$ is the variety determined by the equations $q_{1}=q_{2}=\ldots q_{k}=0$.

We use the above notation also for varieties in projective spaces determined by the homogeneous polynomials $q_{1}, q_{2}, \ldots, q_{k}$.

\subsection{Rank of orbits and proof of first main result}

Each $\sigma \in \mathbf{P}(N)$ is a product of disjoint transpositions. Hence for $\sigma \in \mathbf{P}(N)$, we define the rank of $\sigma$ to be the number of transpositions in $\sigma$. Note that under the one-to-one correspondence between $\mathbf{P}(N)$ and $\mathbf{P M}(N)$, the rank of a permutation is equal to the rank of the corresponding partial permutation matrix.

- $\mathbf{R P}(N)$ denotes the permutations in $\mathbf{P}(N)$ of rank $n$.

Note that all moves other than type I preserve the rank of $\sigma$. Indeed, the only way of obtaining $\sigma$ of smaller rank by applying our moves is by deleting a transposition, which is the effect of move of type I. Also note that it is impossible to have a move of type II or a move of type IV between two permutations in $\mathbf{R P}(N)$. For example, we draw the Hasse diagram for $\mathbf{R P}(6)$ where each dotted line denotes a move of type III and solid line denotes a move of type V (see Fig. 2). Such Hasse diagrams, with particular attention paid to the maximal elements, will lead to the proof of our first main result.

Theorem 2. Conjecture 5 holds for $N<8$.

Proof. Take $N<8, d=\left(d_{1}, d_{2}, \ldots, d_{N}\right)$ an $N$-tuple of nonincreasing integers, and $\psi: \mathbb{P}_{k}^{r-1} \rightarrow V(d)-L(d)$ a nonconstant morphism. Then $\sigma=\sigma_{\psi}$ is in $\mathbf{R P}(N)$. By considering Figs. 1 and 2, we note that there exists a unique maximal $\sigma^{\prime} \in \mathbf{R P}(N)$ such that $\sigma$ can be obtained from $\sigma^{\prime}$ by a sequence of moves of type III. Since moves of type III do not change the number of leading zero rows and ending zero columns of the corresponding partial permutation matrices, the Borel orbit corresponding to $\sigma$ is contained in $V(d)_{\mathcal{R C}}$ if and only if the Borel orbit corresponding to $\sigma^{\prime}$ is contained in $V(d)_{\mathcal{R C}}$ for all $\mathcal{R}, \mathcal{C}$. Hence it is enough to 


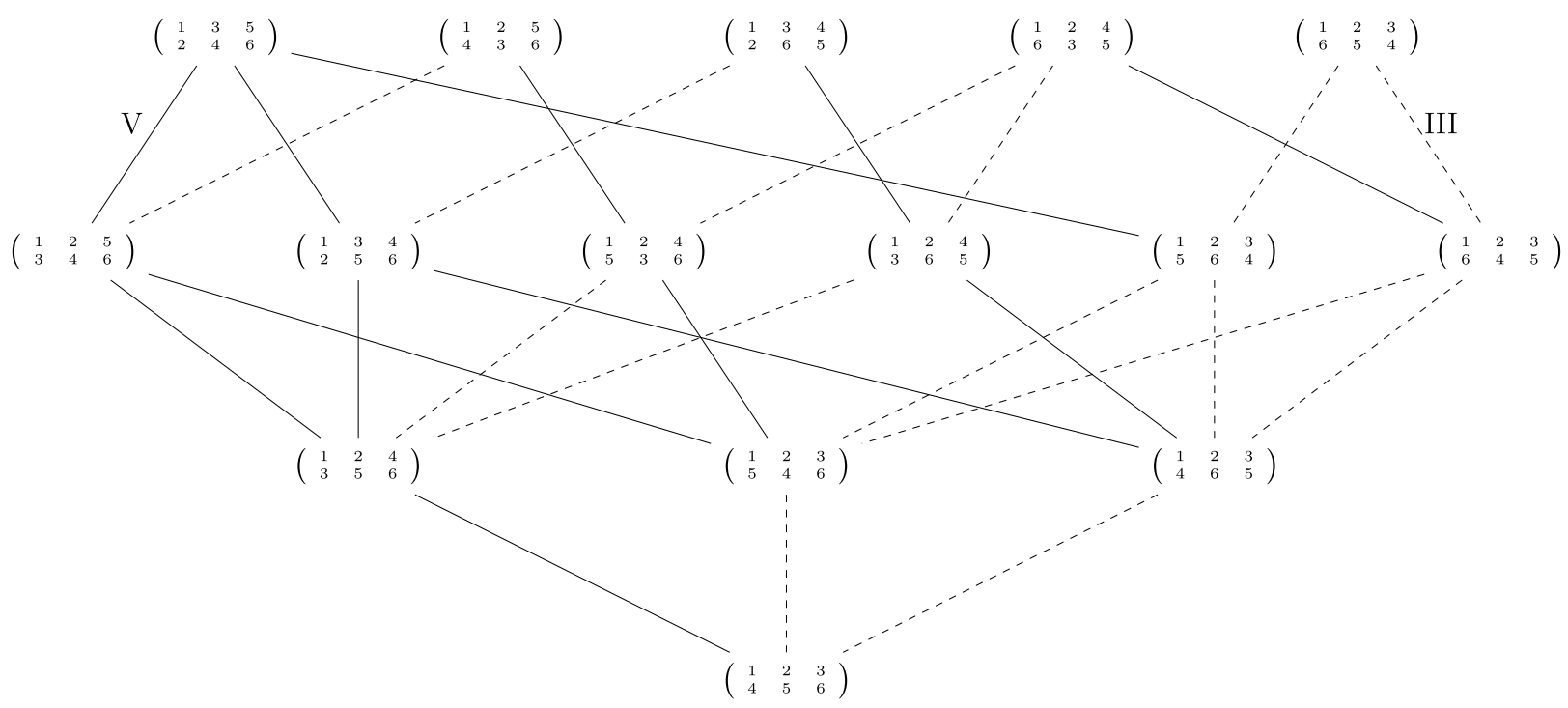

Fig. 2. Hasse diagram of $\mathbf{R P}(6)$.

consider the cases where $\sigma$ is less than or equal to a maximal element in $\mathbf{R P}(N)$ for $N=2,4,6$. We cover these cases by proving in the following eight statements:

(i) If $\sigma=(1,2)$ then $r<2$.

Assume to the contrary that $\sigma=(1,2)$ and $r \geq 2$. If we also write $\psi$ for its restriction to $\mathbb{P}_{k}^{1} \subseteq \mathbb{P}_{k}^{r-1}$, we get a map of the form

$$
\psi(x: y)=\left[\begin{array}{cc}
0 & p_{12} \\
0 & 0
\end{array}\right],
$$

where $p_{12}$ is a homogeneous polynomial in $k[x, y]$. Since $k$ is algebraically closed, there exists $\gamma \in \mathbb{P}_{k}^{1}$ such that $p_{12}(\gamma)=0$. This means $\psi(\gamma)$ is in $L(d)$, which is a contradiction.

(ii) If $\sigma \leq(1,2)(3,4)$ then $r<3$.

Suppose to the contrary that $\sigma \leq(1,2)(3,4)$ and $r \geq 3$. When we restrict $\psi$ to $\mathbb{P}_{k}^{2}$, we get a map of the form

$$
\psi(x: y: z)=\left[\begin{array}{cccc}
0 & p_{12} & p_{13} & p_{14} \\
0 & 0 & 0 & p_{24} \\
0 & 0 & 0 & p_{34} \\
0 & 0 & 0 & 0
\end{array}\right]
$$

Note that there exists $\gamma$ in $\mathbb{P}_{k}^{2}$ such that

$$
p_{12}(\gamma)=0 \text { and } p_{13}(\gamma)=0
$$

Again this means $\psi(\gamma) \in L(d)$. Hence this case is proved by contradiction as well.

(iii) If $\sigma \leq(1,4)(2,3)$ then $r<2$. 
Suppose we have

$$
\psi(x: y)=\left[\begin{array}{cccc}
0 & 0 & p_{13} & p_{14} \\
0 & 0 & p_{23} & p_{24} \\
0 & 0 & 0 & 0 \\
0 & 0 & 0 & 0
\end{array}\right]
$$

Let $m_{j_{1} j_{2} \ldots j_{k}}^{i_{1} i_{2} \ldots i_{k}}$ be as in Section 3.5 and use the same notation to denote its composition with $\psi$. Then there exists $\gamma$ in $\mathbb{P}_{k}^{1}$ such that

$$
m_{34}^{12}(\gamma)=\left(p_{13} p_{24}-p_{23} p_{14}\right)(\gamma)=0
$$

This again gives a contradiction.

(iv) If $\sigma \leq(1,2)(3,4)(5,6)$ then $r<3$.

Suppose otherwise. We have

$$
\psi(x: y: z)=\left[\begin{array}{cccccc}
0 & p_{12} & p_{13} & p_{14} & p_{15} & p_{16} \\
0 & 0 & 0 & p_{24} & p_{25} & p_{26} \\
0 & 0 & 0 & p_{34} & p_{35} & p_{36} \\
0 & 0 & 0 & 0 & 0 & p_{46} \\
0 & 0 & 0 & 0 & 0 & p_{56} \\
0 & 0 & 0 & 0 & 0 & 0
\end{array}\right]
$$

If $p_{12}$ and $p_{13}$ are not relatively prime homogeneous polynomials then there exists $\gamma \in \mathbb{P}_{k}^{2}$ such that

$$
p_{12}(\gamma)=0, p_{13}(\gamma)=0, \text { and } m_{456}^{123}(\gamma)=0
$$

Moreover, if $p_{46}(\gamma)=0$ and $p_{56}(\gamma)=0$, then the rank of $\psi(\gamma)$ is at most 2, which leads to a contradiction. Hence we have $p_{46}(\gamma) \neq 0$ or $p_{56}(\gamma) \neq 0$. Let

$$
c_{4}(\gamma):=\left[\begin{array}{l}
p_{14}(\gamma) \\
p_{24}(\gamma) \\
p_{34}(\gamma)
\end{array}\right] \text { and } c_{5}(\gamma):=\left[\begin{array}{l}
p_{15}(\gamma) \\
p_{25}(\gamma) \\
p_{35}(\gamma)
\end{array}\right]
$$

Since $\psi^{2}=0, c_{4}(\gamma) p_{46}(\gamma)+c_{5}(\gamma) p_{56}(\gamma)=0$. By the fact that $p_{46}(\gamma) \neq 0$ or $p_{56}(\gamma) \neq 0, c_{4}(\gamma)$ and $c_{5}(\gamma)$ are linearly dependent. Thus the rank of $\psi(\gamma)$ is at most 2 , which is a contradiction.

Therefore we may assume $p_{12}$ and $p_{13}$ are relatively prime. Since $\psi^{2}=0$, we have $p_{12} p_{24}+p_{13} p_{34}=0$ and $p_{12} p_{25}+p_{13} p_{35}=0$. This implies that $p_{12}$ divides $p_{34}$ and $p_{35}$, and similarly $p_{13}$ divides $p_{24}$ and $p_{25}$. Then there exists $\gamma$ in $\mathbb{P}_{k}^{2}$ such that

$$
p_{12}(\gamma)=0 \text { and } p_{13}(\gamma)=0
$$

This means $p_{12}, p_{13}, p_{24}, p_{25}, p_{34}$, and $p_{35}$ all vanish at $\gamma$. Hence $\psi(\gamma) \in L(d)$, which is a contradiction.

(v) If $\sigma \leq(1,2)(3,6)(4,5)$ then $r<3$, and (vi) If $\sigma \leq(1,4)(2,3)(5,6)$ then $r<3$. 
These cases are symmetric, so it is enough to prove $(\mathbf{v})$. Consider

$$
\psi(x: y: z)=\left[\begin{array}{cccccc}
0 & p_{12} & p_{13} & p_{14} & p_{15} & p_{16} \\
0 & 0 & 0 & 0 & p_{25} & p_{26} \\
0 & 0 & 0 & 0 & p_{35} & p_{36} \\
0 & 0 & 0 & 0 & p_{45} & p_{46} \\
0 & 0 & 0 & 0 & 0 & 0 \\
0 & 0 & 0 & 0 & 0 & 0
\end{array}\right]
$$

We modify $\psi$ by the operations in Section 3.5. First apply $R_{6,5}(u)$ to $\psi$ for a new variable $u$. If $p_{46}+u p_{45} \neq 0$, apply $D_{5}\left(1 /\left(p_{46}+u p_{45}\right)\right)$ and then $R_{5,6}\left(-p_{45}\right)$ to obtain a matrix of the form

$$
\left[\begin{array}{cccccc}
0 & p_{12} & p_{13} & p_{14} & * & * \\
0 & 0 & 0 & 0 & m_{56}^{24} & p_{26}+u p_{25} \\
0 & 0 & 0 & 0 & m_{56}^{34} & p_{36}+u p_{35} \\
0 & 0 & 0 & 0 & 0 & p_{46}+u p_{45} \\
0 & 0 & 0 & 0 & 0 & 0 \\
0 & 0 & 0 & 0 & 0 & 0
\end{array}\right] .
$$

Hence by selecting a correct value for $u$ we would be done if $V\left(m_{56}^{24}, m_{56}^{34}\right) \nsubseteq V\left(p_{45}\right)$. We may assume

$$
V\left(m_{56}^{24}, m_{56}^{34}\right) \subseteq V\left(p_{45}\right)
$$

Similarly, we may also assume

$$
V\left(m_{56}^{23}, m_{56}^{34}\right) \subseteq V\left(p_{35}\right) \text { and } V\left(m_{56}^{23}, m_{56}^{24}\right) \subseteq V\left(p_{25}\right)
$$

Therefore,

$$
V\left(m_{56}^{23}, m_{56}^{34}, m_{56}^{24}\right) \subseteq V\left(p_{25}, p_{35}, p_{45}\right)=\emptyset
$$

Thus, $\left\{m_{56}^{23}, m_{56}^{34}, m_{56}^{24}\right\}$ is a regular sequence in $k[x, y, z]$. If $p_{45}$ and $p_{46}$ are not relatively prime, there exists $\gamma$ such that $m_{56}^{23}(\gamma)=0$, and $p_{45}(\gamma)=p_{46}(\gamma)=0$. Hence, we may assume $p_{45}$ and $p_{46}$ are relatively prime, which leads a contradiction as we have

$$
p_{45} m_{56}^{23}+p_{25} m_{56}^{34}-p_{35} m_{56}^{24}=0 .
$$

(vii) If $\sigma \leq(1,6)(2,3)(4,5)$ then $r<2$.

To prove this case, consider

$$
\psi(x: y)=\left[\begin{array}{cccccc}
0 & 0 & p_{13} & p_{14} & p_{15} & p_{16} \\
0 & 0 & p_{23} & p_{24} & p_{25} & p_{26} \\
0 & 0 & 0 & 0 & p_{35} & p_{36} \\
0 & 0 & 0 & 0 & p_{45} & p_{46} \\
0 & 0 & 0 & 0 & 0 & 0 \\
0 & 0 & 0 & 0 & 0 & 0
\end{array}\right]
$$

Again by applying $R_{i, j}(u)$ and $D_{i}(v)$ for some $u, v$ we may assume that 


$$
V\left(m_{356}^{124}\right) \subseteq V\left(p_{13}, p_{23}\right) \text { and } V\left(m_{456}^{123}\right) \subseteq V\left(p_{14}, p_{24}\right)
$$

Hence $\left\{m_{356}^{124}, m_{456}^{123}\right\}$ must be a regular sequence in $k[x, y]$. However this is impossible because the determinant of

$$
\left[\begin{array}{ccc}
\operatorname{gcd}\left(p_{13}, p_{14}\right) & p_{15} & p_{16} \\
\operatorname{gcd}\left(p_{23}, p_{24}\right) & p_{25} & p_{26} \\
0 & \operatorname{gcd}\left(p_{35}, p_{45}\right) & \operatorname{gcd}\left(p_{36}, p_{46}\right)
\end{array}\right]
$$

divides both $m_{356}^{124}$ and $m_{456}^{123}$.

(viii) If $\sigma \leq(1,6)(2,5)(3,4)$ then $r<2$.

It is enough to consider a root of $m_{456}^{123}$ to prove this case.

Note that in the above proof the last two cases prove Conjecture 5 when $N \leq 6$ and $r \leq 2$. In the rest of the paper we will generalize these ideas to prove the conjecture for $r \leq 2$. To do this we examine the dimensions of these varieties.

\subsection{Orbit dimensions and proof of second main result}

We now introduce notation for dimensions of these varieties. In this subsection, for $\sigma \in \mathbf{P}(N)$, if the rank of $\sigma$ is $s$, then we obtain two sequences of numbers $i_{1}, \ldots, i_{s}$ and $j_{1}, \ldots, j_{s}$ satisfying the following:

$$
\sigma=\left(i_{1}, j_{1}\right)\left(i_{2}, j_{2}\right) \ldots\left(i_{s}, j_{s}\right)
$$

with $i_{1}<i_{2}<\cdots<i_{s}$ and $i_{a}<j_{a}$ for all $1 \leq a \leq s$. In [18], Melnikov gives a formula for the dimension of a Borel orbit $B_{\sigma}$ for $\sigma$ in $\mathbf{P}(N)$ as follows:

- $f_{t}(\sigma):=\#\left\{j_{p} \mid p<t, j_{p}<j_{t}\right\}+\#\left\{j_{p} \mid p<t, j_{p}<i_{t}\right\}$ for $2 \leq t \leq s$,

- $\operatorname{dim}\left(B_{\sigma}\right)=N s+\sum_{t=1}^{s}\left(i_{t}-j_{t}\right)-\sum_{t=2}^{s} f_{t}(\sigma)$.

We define a new subset of $\mathbf{R P}(N)$ :

- $\mathbf{D P}(N)$ is the set of all $\sigma$ in $\mathbf{R P}(N)$ such that $\operatorname{dim}\left(B_{\sigma^{\prime}}\right)=\operatorname{dim}\left(B_{\sigma}\right)-1$ whenever $\sigma^{\prime}$ is a permutation obtained by applying a single move of type I to $\sigma$.

For instance, the following is the Hasse diagram of $\mathbf{D P}(8)$ (Fig. 3). Note that in the Hasse diagram of $\mathbf{D P}(8)$ all moves are of type $\mathrm{V}$. This is generally the case, which we will prove below. Before we do so, we will prove an easier result that will introduce the notation and argument style that will be necessary.

Fix $\sigma \in \mathbf{D P}(N)$. We use the our convention for $\sigma$ at the beginning of this subsection which implies $i_{1}=1$.

For $q \in\{1, \ldots, n\}$, let $\sigma^{\prime}$ be the result of applying the move of type I that deletes the $q$ th transposition of $\sigma$, so that

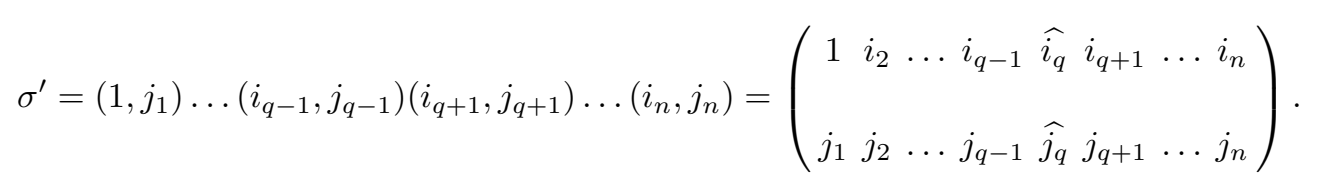




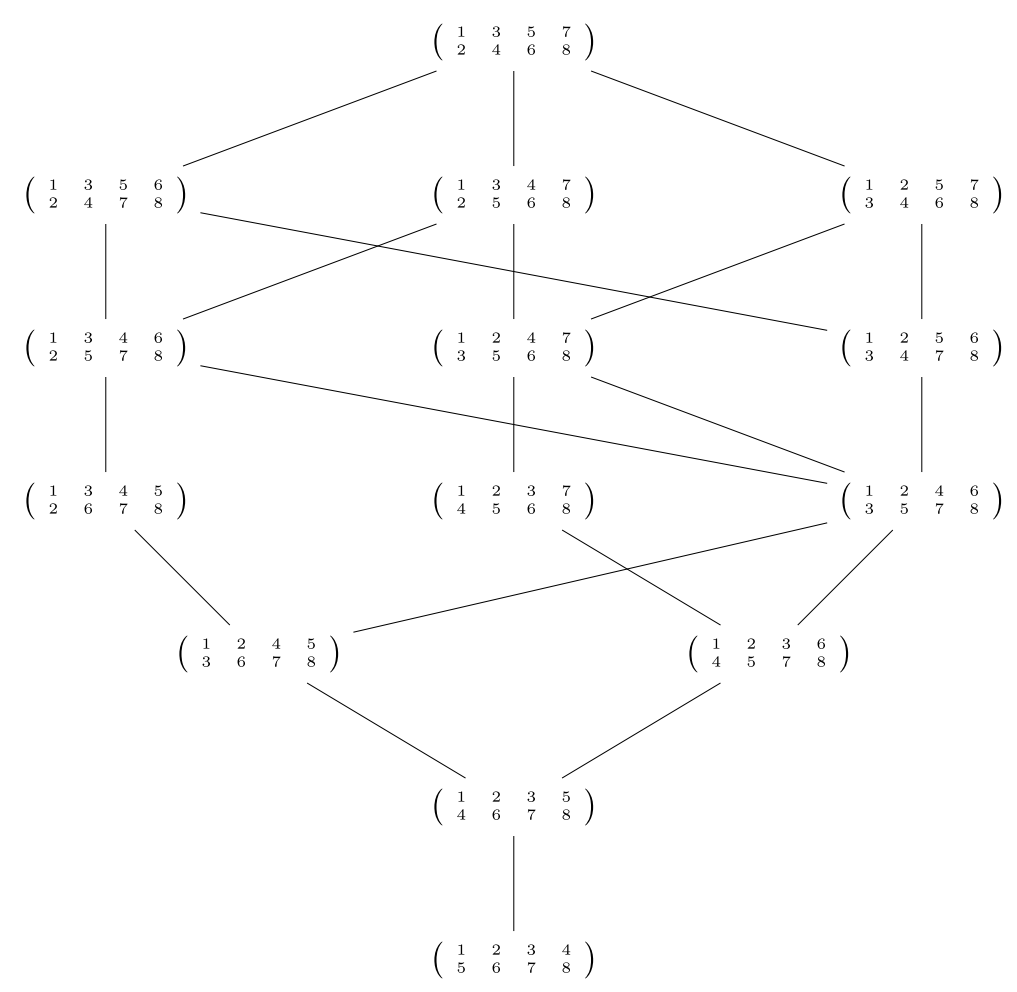

Fig. 3. Hasse diagram of $\mathbf{D P}(8)$.

Then by Melnikov's formula we have

$$
\begin{aligned}
\operatorname{dim}\left(B_{\sigma}\right) & =N n+\sum_{t=1}^{n}\left(i_{t}-j_{t}\right)-\sum_{t=2}^{n} f_{t}(\sigma), \text { and } \\
\operatorname{dim}\left(B_{\sigma^{\prime}}\right) & =N(n-1)+\sum_{t=1}^{n-1}\left(i_{t}-j_{t}\right)-\sum_{t=2}^{n-1} f_{t}\left(\sigma^{\prime}\right) .
\end{aligned}
$$

To simplify our calculation, we write $f_{t}(\sigma)=f_{t}^{1}(\sigma)+f_{t}^{2}(\sigma)$ for $2 \leq t \leq n$, where

$$
f_{t}^{1}(\sigma)=\#\left\{j_{p} \mid p<t, j_{p}<j_{t}\right\} \text { and } f_{t}^{2}(\sigma)=\#\left\{j_{p} \mid p<t, j_{p}<i_{t}\right\}
$$

and we use the notation:

$$
f_{t, q}^{l}\left(\sigma^{\prime}\right)= \begin{cases}f_{t}^{l}\left(\sigma^{\prime}\right) & \text { if } t \leq q-1 \\ 0 & \text { if } t=q \\ f_{t-1}^{l}\left(\sigma^{\prime}\right) & \text { if } t \geq q+1\end{cases}
$$

for $l=1,2$.

Lemma 3. If $N \neq 2$ and the transposition $(1, N)$ appears in $\sigma$, then $\sigma \notin \mathbf{D P}(N)$.

Proof. If $(1, N)$ appears in $\sigma$ and $\sigma \in \mathbf{D P}(N)$, let $q=2$ and $\sigma^{\prime}$ be the result of deleting the second transposition from $\sigma$. Since the $i$ 's are increasing and $i_{a}<j_{a}$ for all $1 \leq a \leq n$, we have $i_{2}=2$, so 


$$
\sigma^{\prime}=\left(\begin{array}{ccccc}
1 & \widehat{2} & i_{3} & \ldots & i_{n} \\
& & & & \\
N & \widehat{j_{2}} & j_{3} & \ldots & j_{n}
\end{array}\right)
$$

Thus $3 \leq j_{2} \leq N-1$. Let $j_{2}=N-b$ for some $1 \leq b \leq N-3$. Then

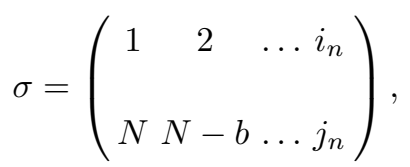

and any number between $N-b$ and $N$ has to appear as a $j$ or an $i$ that is bigger than $j_{2}$. Therefore,

$$
\left(\sum_{t=2}^{n} f_{t}^{1}(\sigma)-f_{t, 2}^{1}\left(\sigma^{\prime}\right)\right)+\left(\sum_{t=2}^{n} f_{t}^{2}(\sigma)-f_{t, 2}^{2}\left(\sigma^{\prime}\right)\right)=b-1 .
$$

Hence, $\operatorname{dim}\left(B_{\sigma}\right)-\operatorname{dim}\left(B_{\sigma^{\prime}}\right)=N+2-N+b-(b-1)=3$, so $\sigma \notin \mathbf{D P}(N)$.

Now we prove the main proposition of this subsection.

Proposition 4. If $\sigma \in \mathbf{D P}(N)$, then $j_{p}<j_{t}$ for all $p<t$, and therefore we cannot apply a move of type III to $\sigma$. Conversely, if $\sigma \in \mathbf{R P}(N)$ and we cannot apply a move of type of III to $\sigma$, then $\sigma \in \mathbf{D P}(N)$.

Proof. Assume that $\sigma \in \mathbf{D P}(N)$. We will prove the following statement by induction on $k$ :

$$
j_{n-k}<\ldots<j_{n-1}<j_{n} \text { and } \forall(p<n-k), j_{p}<j_{n-k} .
$$

Suppose $k=0$. To prove $(*)$, we need to show that $\forall p<n, j_{p}<j_{n}$. Let $\sigma^{\prime}$ be obtained by deleting $n$th transposition of $\sigma$.

$$
\sigma^{\prime}=\left(\begin{array}{ccccc}
1 & i_{2} & \ldots & i_{n-1} & \widehat{i_{n}} \\
& & & & \\
j_{1} & j_{2} & \ldots & j_{n-1} & \widehat{j_{n}}
\end{array}\right)
$$

Since $\sigma \in \mathbf{D P}(N)$, we have

$$
1=\operatorname{dim}\left(B_{\sigma}\right)-\operatorname{dim}\left(B_{\sigma^{\prime}}\right)=N+\left(i_{n}-j_{n}\right)-\left(f_{n}^{1}(\sigma)+f_{n}^{2}(\sigma)\right)
$$

Since the total number of possible $j$ except $j_{n}$ is $n-1$, and any number between $i_{n}$ and $j_{n}$ has to appear as $j$, we have $f_{n}^{2}(\sigma)=n-1-\left(j_{n}-i_{n}-1\right)$. By the equation $(1), f_{n}^{1}(\sigma)=n-1$, so that $\forall p<n, j_{p}<j_{n}$ is true. Therefore $j_{n}=N$.

Now assume the statement $(*)$ is true for $k$. Then we can visualise $\sigma$ as follows:

$$
\sigma=\left(\begin{array}{cccccc}
1<i_{2} & <\ldots & <i_{n-k-1}<i_{n-k} & <i_{n-k+1}<\ldots<i_{n} \\
j_{1} & j_{2} & \ldots & j_{n-k-1} & j_{n-k}<j_{n-k+1}<\ldots<j_{n}
\end{array}\right)
$$

We need to prove $(*)$ for $k+1$, that is,

$$
j_{n-k-1}<\ldots<j_{n-1}<j_{n} \text { and } \forall(p<n-k-1), j_{p}<j_{n-k-1} \text {. }
$$


By the second part of the inductive hypothesis for $k$, we have $j_{n-k-1}<j_{n-k}$ so the first part of $(*)$ is already true, and we only need to show that the second part holds. In other words, it is enough to show that $f_{n-k-1}^{1}(\sigma)=n-k-2$. Let $\sigma^{\prime}$ be obtained by deleting $(n-k-1)$ th transposition of $\sigma$. Then we have

$$
\sum_{t=n-k}^{n} f_{t}^{1}(\sigma)-f_{t, n-k-1}^{1}\left(\sigma^{\prime}\right)=k+1
$$

Let $w:=\#\left\{i_{p} \mid i_{n-k-1}<i_{p}<j_{n-k-1}\right\}$. Then

$$
f_{n-k-1}^{2}(\sigma)=n-(k+2)-\left(j_{n-k-1}-i_{n-k-1}-1-w\right)
$$

and

$$
\sum_{t=n-k}^{n} f_{t}^{2}(\sigma)-f_{t, n-k-1}^{2}\left(\sigma^{\prime}\right)=k+1-w
$$

By the fact that $\operatorname{dim}\left(B_{\sigma}\right)-\operatorname{dim}\left(B_{\sigma^{\prime}}\right)=1$, we have $f_{n-k-1}^{1}(\sigma)=n-k-2$. Thus the first claim is proved.

Conversely, given $\sigma \in \mathbf{R P}(N)$, suppose that $\sigma^{\prime}$ is the result of applying the move of type I that deletes the $q$-th transposition from $\sigma$. Note that $f_{q}^{1}(\sigma)=q-1$ and $\sum_{t=q+1}^{n} f_{t}^{1}(\sigma)-f_{t, q}^{1}\left(\sigma^{\prime}\right)=n-q$. Hence,

$$
\sum_{t=2}^{n} f_{t}^{1}(\sigma)-f_{t, q}^{1}\left(\sigma^{\prime}\right)=n-1
$$

Then,

$$
\operatorname{dim}\left(B_{\sigma}\right)-\operatorname{dim}\left(B_{\sigma^{\prime}}\right)=N+\left(i_{q}-j_{q}\right)-(n-1)-\left(\sum_{t=2}^{n} f_{t}^{2}(\sigma)-f_{t, q}^{2}\left(\sigma^{\prime}\right)\right)
$$

We also have the difference $f_{t}^{2}(\sigma)-f_{t}^{2}\left(\sigma^{\prime}\right)=0$ when $t \in\{1, \ldots, q-1\}$. Therefore,

$$
\begin{aligned}
\sum_{t=2}^{n} f_{t}^{2}(\sigma)-f_{t, q}^{2}\left(\sigma^{\prime}\right)= & \sum_{t=q}^{n} \#\left\{j_{p} \mid p<t, j_{p}<i_{t}\right\} \\
& -\sum_{t=q+1}^{n} \#\left\{j_{p} \mid p<t, p \neq q, j_{p}<i_{t}\right\} \\
= & \#\left\{j_{p} \mid j_{p}<i_{q}\right\}+\#\left\{i_{t} \mid j_{q}<i_{t}\right\} .
\end{aligned}
$$

Let $F=\#\left\{j_{p} \mid j_{p}<i_{q}\right\}$ and $G=\#\left\{i_{t} \mid j_{q}<i_{t}\right\}$. Note that numbers between $i_{q}$ and $j_{q}$ must appear as $j_{l}$ for $l<q$ or as $i_{s}$ where $s>q$. Let $a=\#\left\{j_{p} \mid i_{q}<j_{p}<j_{q}\right\}$ and $b=\#\left\{i_{t} \mid i_{q}<i_{t}<j_{q}\right\}$. Then $a+b=j_{q}-i_{q}-1$. Let $A=\#\left\{j_{p} \mid i_{q}<j_{p}\right\}$ and $B=\#\left\{i_{t} \mid i_{t}<j_{q}\right\}$. We have $A-a+B-b-1=n$. Therefore, $A+B=n+j_{q}-i_{q}$.

$$
\sigma=\left(\begin{array}{l}
\overbrace{i_{1}, \ldots \ldots, i_{q} \underbrace{}_{\mathrm{b}} \ldots, i_{n}}^{\mathrm{B}} \overbrace{\mathrm{F}}^{\mathrm{G}} \\
\underbrace{\mathrm{B}}_{\mathrm{A}} \underbrace{j_{1}, \ldots}_{\overbrace{j_{q}, \ldots \ldots, j_{n}}, \ldots, \ldots}
\end{array}\right)
$$


Since $\sigma \in \mathbf{R P}(N), A+B+F+G=2 n$. Then $F+G=n-j_{q}+i_{q}$. Consequently, $\operatorname{dim}\left(B_{\sigma}\right)-\operatorname{dim}\left(B_{\sigma^{\prime}}\right)=1$, so $\sigma \in \mathbf{D P}(N)$.

Lemma 5. For every $X$ in $V(d)-L(d)$ we have

$$
r_{i j}(X) \geq j-i+1-n
$$

Proof. The rank of $X$ is $n$, so $r_{1 N}(X)=n$. The result follows from the inequality

$$
r_{i j}(X)+(i-1)+(N-j) \geq r_{1 N}(X)
$$

We now define our last set of permutations:

- $\mathbf{M P}(r, N)$ is the set of minimal permutations in $\mathbf{P}(N)$ that appear as a permutation in the form $\sigma_{\psi}$ for some $d$ and nonconstant morphism $\psi: \mathbb{P}_{k}^{r-1} \rightarrow V(d)-L(d)$.

We now state and prove our second main result.

Theorem 6. Conjecture 5 holds for $r \leq 2$.

Proof. We have $\mathbf{M P}(1, N)=\{(1, n+1)(2, n+2) \ldots(n, N)\}$ (see Example 1$)$. This means Conjecture 5 holds for $r=1$, because $N-n+(n+1)-1=N \leq N$. Hence it is enough to prove Conjecture 5 for $r=2$.

Suppose that Conjecture 5 does not hold for $r=2$. Then there exists an $N$-tuple of nonincreasing integers $d=\left(d_{1}, d_{2}, \ldots, d_{N}\right)$, two positive integers $\mathcal{R}, \mathcal{C}$, and a nonconstant morphism $\psi: \mathbb{P}_{k}^{1} \rightarrow V(d)_{\mathcal{R C}}-L(d)$ such that $N<2(\mathcal{R}+\mathcal{C})$, or equivalently $n<\mathcal{R}+\mathcal{C}$. Write $\sigma_{\psi}=\left(i_{1}, j_{1}\right)\left(i_{2}, j_{2}\right) \ldots\left(i_{n}, j_{n}\right)$ with $1=i_{1}<i_{2}<$ $\cdots<i_{n}$ and $i_{a}<j_{a}$ for all $1 \leq a \leq n$.

First assume that $\sigma \in \mathbf{D P}(N)$. By Proposition 4, we have $j_{1}<j_{2}<\cdots<j_{n}=N$. Therefore, $\mathcal{C}=j_{1}-1$ and $\mathcal{R}=N-i_{n}$. Moreover, for every $a$ we have $j_{a}>\mathcal{C}$ and $i_{a}<N-\mathcal{R}+1$. Set $I:=\left\{i_{1}, \ldots, i_{n}\right\}$. Note that $\{1, \ldots, \mathcal{C}\} \subseteq I$ since $\forall a, j_{a}>\mathcal{C}$. So, $i_{a}=a$ if $a \leq \mathcal{C}$. Similarly, $j_{a-n}=a$ if $a \geq N-\mathcal{R}+1$. Set

$$
\underline{i}:=n-\mathcal{R}+1 \text { and } \bar{i}:=\mathcal{C}
$$

and

$$
\underline{j}:=N-\mathcal{R}+1 \text { and } \bar{j}:=\mathcal{C}+n
$$

We have

$$
\bar{i}-\underline{i}=\mathcal{R}+\mathcal{C}-n-1=\bar{j}-\underline{j} .
$$

In particular, $\underline{i} \leq \bar{i}$ and $\underline{j} \leq \bar{j}$, since we assumed that $n<\mathcal{R}+\mathcal{C}$.

For $i \leq i^{\prime}$ and $j \leq j^{\prime}$, let $A_{j, j^{\prime}}^{i, i^{\prime}}$ denote the submatrix of the partial permutation matrix $P_{\sigma}$ obtained by considering the rows from $i$ to $i^{\prime}$ and columns from $j$ to $j^{\prime}$. Note that $A_{1, N}^{1, \bar{i}}$ has $\mathcal{C}$ many 1 's and $A_{\underline{j}, N}^{1, N}$ has $\mathcal{R}$ many 1 's. Hence $A_{\underline{j}, N}^{1, \bar{i}}$ must have $(\mathcal{R}+\mathcal{C}-n)$ many 1 's. However, there is no 1 in $A_{\underline{j}, N}^{1, i-1}$; otherwise there would exist $a$ such that $i_{a}<\underline{i}=n-\mathcal{R}+1$ and $j_{a} \geq \underline{j}$, which leads to a contradiction by considering the number of 1's in the region determined by the union of $A_{1, N}^{1, i_{a}-1}$ and $A_{j_{a}+1, N}^{1, N}$. Similarly, there is no 1 in $A_{\bar{j}+1, N}^{1, \bar{i}}$. Hence the $(\mathcal{R}+\mathcal{C}-n) \times(\mathcal{R}+\mathcal{C}-n)$-submatrix $A_{\underline{j}, \bar{j}}^{\underline{i}, \bar{i}}$ contains $(\mathcal{R}+\mathcal{C}-n)$ many $1^{\prime}$ s. Thus, $A_{\underline{j}, \bar{j}}^{\underline{i}, \bar{i}}$ is the identity matrix of dimension $\mathcal{R}+\mathcal{C}-n$. 


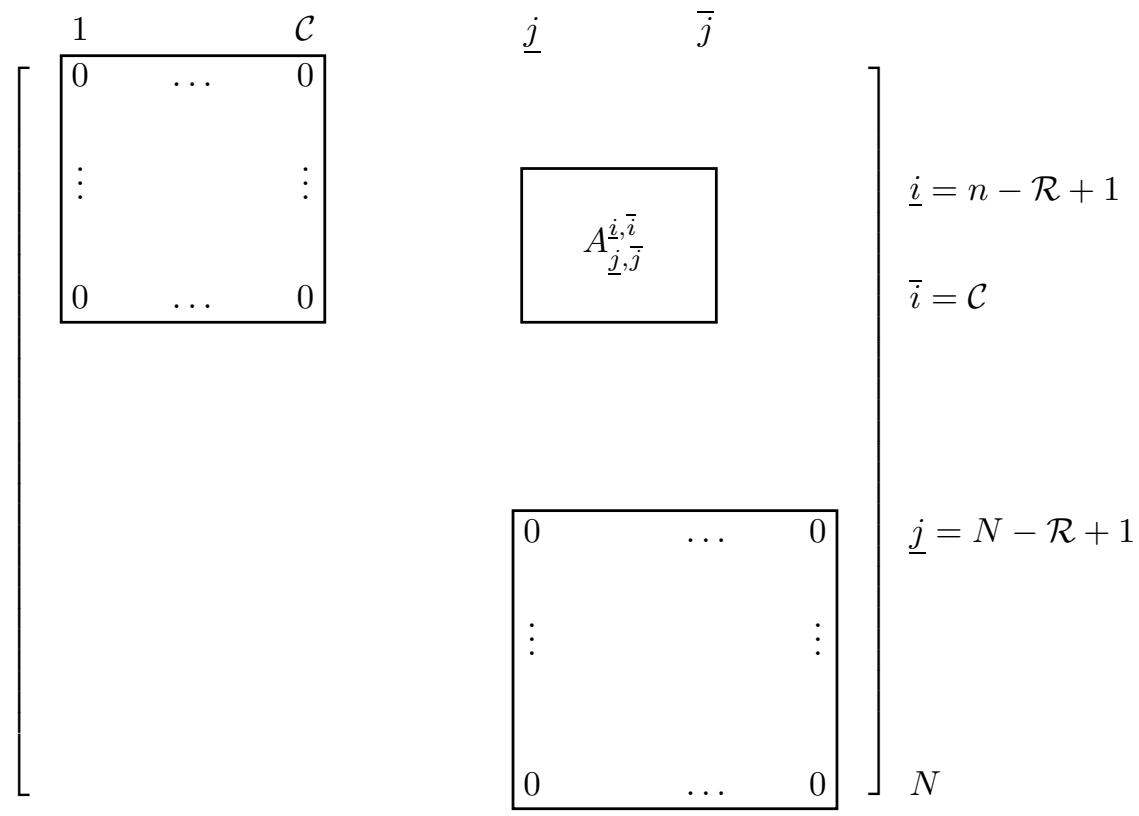

This in particular means that for every $X$ in the image of $\psi$ we have

$$
r_{\underline{i}, \underline{j}-1}(X)=r_{n-\mathcal{R}+1, N-\mathcal{R}}(X)=0
$$

and

$$
r_{\bar{i}+1, \bar{j}}(X)=r_{\mathcal{C}+1, \mathcal{C}+n}(X)=0 .
$$

Since $k$ is algebraically closed, there exists a root of the minor $m_{\underline{j}, \underline{i}, \underline{j}+1 \ldots, \bar{i}}^{\underline{i}+1 \ldots, \bar{j}}$. Thus, there exists $X$ in the image of $\psi$ such that

$$
r_{\underline{i}, \bar{j}}(X) \leq \bar{i}-\underline{i}
$$

which means

$$
r_{n-\mathcal{R}+1, \mathcal{C}+n}(X) \leq \mathcal{R}+\mathcal{C}-n-1 .
$$

Lemma 5 implies that for every $X$ in the image of $\psi$ we have

$$
r_{n-\mathcal{R}+1, \mathcal{C}+n}(X) \geq \mathcal{C}+n-(n-\mathcal{R}+1)+1-n=\mathcal{R}+\mathcal{C}-n .
$$

This is a contradiction, so we are done with the case $\sigma \in \mathbf{D P}(N)$.

Now assume that $\sigma \notin \mathbf{D P}(N)$. We recursively define perturbations of $\psi$ so that we can again use the square submatrix $A_{\underline{\underline{j}}, \bar{j}}^{\underline{i}, \bar{i}}$ to get a contradiction similar to that of the previous case. Set $\psi^{0}=\psi, n_{0}=0$, $Z_{0}=\emptyset$. We have a rational map $\psi^{0}: \mathbb{A}_{k}^{2+n_{0}}-Z_{0} \rightarrow V_{N}$. Now given

$$
\psi^{s}: \mathbb{A}_{k}^{2+n_{s}}-Z_{s} \rightarrow V_{N}
$$

we define $\psi^{s+1}: \mathbb{A}_{k}^{2+n_{s+1}}-Z_{s+1} \rightarrow V_{N}$ when $\sigma_{s}=\sigma_{\psi^{s}}$ is not in $\mathbf{D P}(N)$. 
Assume $\sigma_{s} \notin \mathbf{D P}(N)$. By Proposition 4 , there exists a move of type III that we can apply to $\sigma_{s}$. Hence, we may define

$$
l_{s}^{\prime}:=\min \left\{l^{\prime} \mid l^{\prime}<l<\sigma_{s}(l)<\sigma_{s}\left(l^{\prime}\right)\right\}
$$

and

$$
l_{s}:=\sigma_{s}\left(\min \left\{\sigma_{s}(l) \mid l_{s}^{\prime}<l<\sigma_{s}(l)<\sigma_{s}\left(l_{s}^{\prime}\right)\right\}\right)
$$

In case $l_{s}^{\prime}<\underline{i}$, we define

$$
n_{s+1}:=n_{s}+l_{s}-l_{s}^{\prime}+1
$$

Note that $l_{s}>l_{s}^{\prime}$ and so $n_{s+1} \geq n_{s}+2$. Hence the affine variety $Z_{s}$ can be considered as a subvariety of $\mathbb{A}_{k}^{2+n_{s+1}}$ by considering $\mathbb{A}_{k}^{2+n_{s}} \subset \mathbb{A}_{k}^{2+n_{s+1}}$. Here we write $\left(x, y, u_{1}, u_{2}, \ldots u_{n_{s+1}}\right)$ to denote a point in $\mathbb{A}_{k}^{2+n_{s+1}}$. Hence $\mathbb{A}_{k}^{2+n_{s}}$ corresponds to the points where $u_{n_{s}+1}=\ldots=u_{n_{s+1}}=0$.

Represent $\psi^{s}$ by a matrix $\left(\psi_{i j}^{s}\right)$ whose $(i, j)$-entry $\psi_{i, j}^{s}$ is a rational function in $k\left(x, y, u_{1}, u_{2}, \ldots u_{n_{s}}\right)$. Let $p_{i}$ denote the entry $\psi_{i, \sigma\left(l_{s}\right)}^{s}$ which is a polynomial. Also let $\bar{p}$ be the greatest common divisor of $p_{l_{s}^{\prime}}, p_{l_{s}^{\prime}+1}, \ldots, p_{l_{s}}$. Set $p_{i}^{\prime}:=p_{i} / \bar{p}$ for $i \in\left\{l_{s}^{\prime}, l_{s}^{\prime}+1, \ldots, l_{s}\right\}$. We define

$$
Z_{s+1}=Z_{s} \cup V\left(u_{n_{s}+1}, \sum_{i:=l_{s}^{\prime}}^{l_{s}} u_{n_{s}+i-l_{s}^{\prime}+1} p_{i}^{\prime}\right)
$$

We obtain $\psi^{s+1}$ from $\psi^{s}$ by first applying $D_{l_{s}^{\prime}}\left(u_{n_{s}+1}\right)$, then $R_{i, l_{s}^{\prime}}\left(u_{i}\right)$ where $l_{s}^{\prime}<i \leq l_{s}$, then $D_{i}\left(\sum_{i:=l_{s}^{\prime}}^{l_{s}} u_{n_{s}+i-l_{s}^{\prime}+1} p_{i}^{\prime}\right)$ for $l_{s}^{\prime}<i \leq l_{s}$, and finally applying $R_{l_{s}^{\prime}, i}\left(-p_{i}^{\prime}\right)$ for $l_{s}^{\prime}<i \leq l_{s}$. Notice that $p_{i}^{\prime}$ also depends on $s$, so we write $p_{s, i}^{\prime}$ instead of $p_{i}^{\prime}$ when $s$ is not clear.

We can repeat this process until it is no longer possible to find a move of type III with $l_{s}^{\prime}<\underline{i}$. At the end of this part of the process we obtain a rational map $\psi^{t}$ for some $t$. Then we can continue with the symmetric (with respect to the diagonal of the matrix running from the lower left entry to the upper right entry) operations assuming $\psi^{s}$ is defined for $s \geq t$. We define $\psi^{s+1}$ as follows:

$$
l_{s}^{\prime}:=\max \left\{l^{\prime} \mid l^{\prime}<l<\sigma_{s}(l)<\sigma_{s}\left(l^{\prime}\right)\right\}
$$

and

$$
l_{s}:=\sigma_{s}\left(\max \left\{\sigma_{s}(l) \mid l_{s}^{\prime}<l<\sigma_{s}(l)<\sigma_{s}\left(l_{s}^{\prime}\right)\right\}\right) .
$$

We repeat the symmetric operations as long as we have $\sigma_{s}\left(l_{s}^{\prime}\right)>\bar{j}$. We define $n_{s+1}:=n_{s}+\sigma_{s}\left(l_{s}^{\prime}\right)-$ $\sigma_{s}\left(l_{s}\right)+1$. Let $p_{j}$ denote the entry $\psi_{l_{s}, j}^{s}$ which is a polynomial. Also let $\bar{q}$ be the greatest common divisor of $p_{\sigma_{s}\left(l_{s}\right)}, p_{\sigma\left(l_{s}\right)+1}, \ldots, p_{\sigma_{s}\left(l_{s}^{\prime}\right)}$. Similarly, we write $p_{s, j}^{\prime}$ instead of $p_{j}^{\prime}$ when $s$ is not clear.

At the end of this process we obtain a rational map $\psi^{\bar{t}}$ from the quasi affine variety $U=\mathbb{A}_{k}^{2+n_{\bar{t}}}-Z_{\bar{t}}$ to $V_{N}$ where we have $r_{\underline{i}, \underline{j}-1}(X)=r_{n-\mathcal{R}+1, N-\mathcal{R}}(X)=0$ and $r_{\bar{i}+1, \bar{j}}(X)=r_{\mathcal{C}+1, \mathcal{C}+n}(X)=0$ for every $X$ in the image of this rational map. Denote the composition of $m_{\underline{j}}^{\underline{i}, \ldots, \ldots \bar{i}}$ with $\psi^{\bar{t}}$ by $m$. Notice that $m$ is a polynomial in $k\left[x, y, u_{1}, \ldots u_{n_{\bar{t}}}\right]$. Define another polynomial $p$ in the same polynomial algebra as follows:

$$
p=\left(\prod_{s=0}^{t-1} u_{n_{s}+1} \sum_{i=l_{s}^{\prime}}^{l_{s}} u_{n_{s}+i-l_{s}^{\prime}+1} p_{s, i}^{\prime}\right)\left(\prod_{s=t}^{\bar{t}-1} u_{n_{s}+1} \sum_{j=\sigma\left(l_{s}\right)}^{\sigma\left(l_{s}^{\prime}\right)} u_{n_{s}+\sigma\left(l_{s}^{\prime}\right)-j+1} p_{s, j}^{\prime}\right) .
$$


Notice $p_{s, l_{s}^{\prime}}^{\prime}, p_{s, l_{s}^{\prime}+1}^{\prime}, \ldots, p_{s, l_{s}}^{\prime}$ are relatively prime for $1 \leq s \leq t$ and similarly $p_{s, \sigma\left(l_{s}\right)}^{\prime}, p_{s, \sigma\left(l_{s}\right)+1}^{\prime}, \ldots, p_{s, \sigma\left(l_{s}^{\prime}\right)}^{\prime}$ are also relatively prime for $t+1 \leq s \leq \bar{t}$. Moreover, the polynomial $m$ has an irreducible factor in $k[x, y]$ or for some $s$, the polynomial $m$ has an irreducible factor in the form $f u_{n_{s}}+g$, where $f$ and $g$ are in $k\left[x, y, u_{1}, \ldots u_{n_{s}-1}\right]$ so that $f$ is neither an associate of $p_{s, l_{s}}^{\prime}$ nor $p_{s, \sigma\left(l_{s}\right)}^{\prime}$. Hence, there exists a solution to the equations $p=1$ and $m=0$, which is again a contradiction by Lemma 5 .

\section{Examples and problems}

The last inequality in Conjecture 5 is equivalent to

$$
r \leq\left\lfloor\log _{2}\left(\frac{N}{\mathcal{R}+\mathcal{C}}\right)\right\rfloor+1
$$

One might ask how strict this upper bound for $r$ is.

In all the following examples, we define $\psi$ from $\mathbb{P}_{k}^{r-1}$ to $V(d)_{\mathcal{R C}}-L(d)$ for different values of $r, N$ and $\mathcal{R}+\mathcal{C}$ where $r=\left\lfloor\log _{2}\left(\frac{N}{\mathcal{R}+\mathcal{C}}\right)\right\rfloor+1$. It follows that we do not have a better upper bound for $r$ in these cases.

Example 1. For $r=1, N=2 n, d=(0, \ldots, 0)$ and $\mathcal{R}+\mathcal{C}=N$, define

$$
\psi(x)=\left[\begin{array}{l|l}
0 & M \\
\hline 0 & 0
\end{array}\right] \text { where } M=\left[\begin{array}{lll}
x & & 0 \\
& \ddots & \\
0 & & x
\end{array}\right] .
$$

Note that $\sigma_{\psi}=(1, n+1)(2, n+2) \ldots(n, N)$. This example shows that

$$
\operatorname{MP}(1, N)=\{(1, n+1)(2, n+2) \ldots(n, N)\} .
$$

Example 2. For $r=2$ and $N=4, d=(0,0,0,0)$ and $\mathcal{R}+\mathcal{C}=2$, define

$$
\psi(x, y)=\left[\begin{array}{rrrr}
0 & x & y & 0 \\
0 & 0 & 0 & y \\
0 & 0 & 0 & -x \\
0 & 0 & 0 & 0
\end{array}\right]
$$

In this example, $\sigma_{\psi}=(1,2)(3,4)$. Hence,

$$
\mathbf{M P}(2,4)=\{(1,2)(3,4)\}
$$

Example 3. For $r=2, N=6, d=(0,-1,-1,-1,-1,-1)$, and $\mathcal{R}+\mathcal{C}=3$, set:

$$
\psi(x, y)=\left[\begin{array}{rrrrrr}
0 & x^{2} & x y & y^{2} & 0 & 0 \\
0 & 0 & 0 & 0 & y & 0 \\
0 & 0 & 0 & 0 & -x & y \\
0 & 0 & 0 & 0 & 0 & -x \\
0 & 0 & 0 & 0 & 0 & 0 \\
0 & 0 & 0 & 0 & 0 & 0
\end{array}\right]
$$


Here, we have $\sigma_{\psi}=(1,2)(3,5)(4,6)$. Considering the Hasse diagram for $\mathbf{R P}(6)$ in Fig. 2 and symmetry it is clear that

$$
\mathbf{M P}(2,6)=\{(1,2)(3,5)(4,6),(1,3)(2,4)(5,6)\}
$$

The above example can be generalized:

Example 4. For $r=2, N=2 n, d=(0,-n+2, \ldots,-n+2)$, and $\mathcal{R}+\mathcal{C}=n$, set:

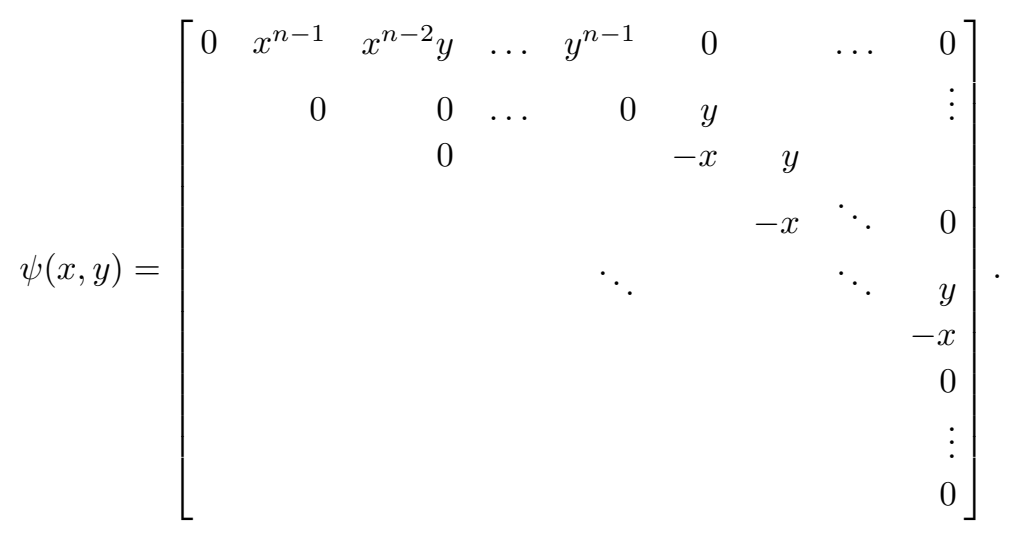

We can use the above examples to obtain new ones by the chess board construction:

Construction 1. Let $\left(l_{1}, l_{2}, \ldots, l_{m}\right)$ be an $m$-tuple of positive integers and $V(d)_{\left(l_{1}, l_{2}, \ldots, l_{m}\right)}$ the subvariety of $V(d)$ such that $x_{i j}=0$ when $l_{1}+l_{2}+\ldots+l_{(s-1)}+1 \leq i<j \leq l_{1}+l_{2}+\ldots+l_{s}$ for some $1 \leq s \leq m$. For example, the following matrix $\psi(x, y)$ is in $V(d)_{(1,3,2)}$ where $d$ is 6-tuple of nonincreasing integers:

$$
\psi(x, y)=\left[\begin{array}{cccccc}
0 & x^{2} & x y & y^{2} & 0 & 0 \\
0 & 0 & 0 & 0 & y & 0 \\
0 & 0 & 0 & 0 & -x & y \\
0 & 0 & 0 & 0 & 0 & -x \\
0 & 0 & 0 & 0 & 0 & 0 \\
0 & 0 & 0 & 0 & 0 & 0
\end{array}\right] .
$$

Take $\psi_{1} \in V\left(d_{1}\right)_{\left(l_{1}^{1}, l_{2}^{1}, \ldots, l_{m}^{1}\right)}$ where $d_{1}$ is $N_{1}$-tuple nonpositive integers and $\psi_{2} \in V\left(d_{2}\right)_{\left(l_{1}^{2}, l_{2}^{2}, \ldots, l_{m}^{2}\right)}$ where $d_{2}$ is $N_{2}$-tuple of nonincreasing integers. We arrange a $\left(N_{1}+N_{2}\right) \times\left(N_{1}+N_{2}\right)$ matrix in a $2 m \times 2 m$-chessboard as follows: The $i j$-square contains a $l_{\left\lfloor\frac{i+1}{2}\right\rfloor}^{\varepsilon_{i}} \times l_{\left\lfloor\frac{j+1}{2}\right\rfloor}^{\varepsilon_{j}}$ matrix such that $\varepsilon_{k}=1$ if $k$ is odd or $\varepsilon_{k}=2$ if $k$ is even integer. Now we color the $i j$ square black if $\varepsilon_{i} \neq \varepsilon_{j}$ and white if $\varepsilon_{i}=\varepsilon_{j}$. Fill in the $i j$ square with zeros if it is a black square and otherwise fill it in with $\left(x_{i^{\prime} j^{\prime}}\right)$ where $\underline{i} \leq i^{\prime} \leq \bar{i}$ and $\underline{j} \leq j^{\prime} \leq \bar{j}$ part of $\psi_{\varepsilon_{i}}$ where $\underline{i}, \bar{i}, \underline{j}, \bar{j}$ are defined by

$$
\underline{s}=\sum_{m=1}^{\left\lfloor\frac{s+1}{2}\right\rfloor-1} l_{m}^{\varepsilon_{s}}+1 \text { and } \bar{s}=\sum_{m=1}^{\left\lfloor\frac{s+1}{2}\right\rfloor} l_{m}^{\varepsilon_{s}} .
$$

For instance, using chessboard construction we can obtain an example:

Example 5. For $r=2, N=4+6=10, d=(0,0,-1,-1,-1,-1,-1,-1,-1,-1)$, and $\mathcal{R}+\mathcal{C}=3+2=5$ we obtain an example by applying the chess board construction on the morphisms in Examples 2 and 3. 


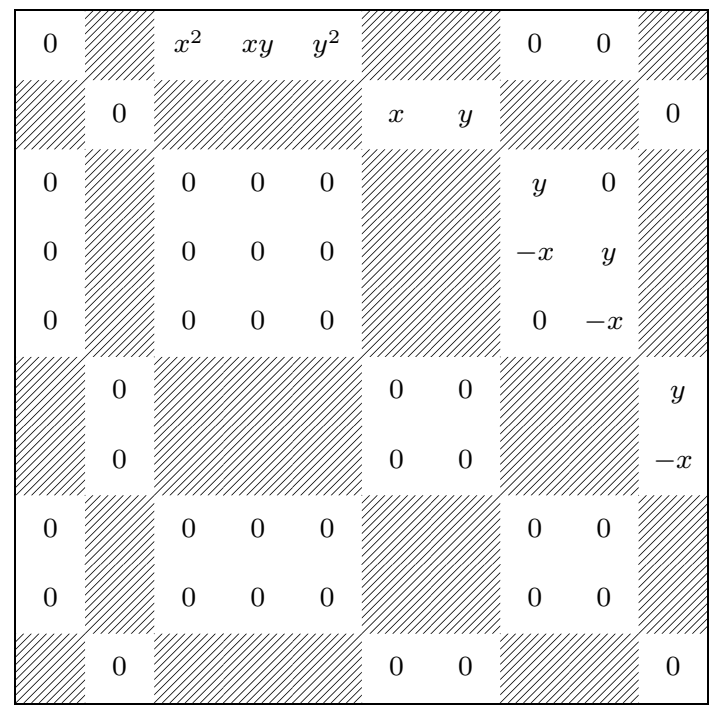

We also have other well-known constructions like the Koszul complex construction [4] giving us examples as below.

Example 6. For $r=3, N=8$ and $\mathcal{R}+\mathcal{C}=2$, define

$$
\psi(x, y, z)=\left[\begin{array}{rrrrrrrr}
0 & x & y & z & 0 & 0 & 0 & 0 \\
0 & 0 & 0 & 0 & y & -z & 0 & 0 \\
0 & 0 & 0 & 0 & -x & 0 & z & 0 \\
0 & 0 & 0 & 0 & 0 & x & -y & 0 \\
0 & 0 & 0 & 0 & 0 & 0 & 0 & z \\
0 & 0 & 0 & 0 & 0 & 0 & 0 & y \\
0 & 0 & 0 & 0 & 0 & 0 & 0 & x \\
0 & 0 & 0 & 0 & 0 & 0 & 0 & 0
\end{array}\right] .
$$

In this example, $\sigma_{\psi}=(1,2)(3,5)(4,6)(7,8)$.

We end with a few questions for future research. Notice that all examples discussed above are in $\mathbf{D P}(N)$. Hence one can ask:

Question 1. Is $\mathbf{M P}(r, N) \subseteq \mathbf{D P}(N)$ ?

For all these examples $\frac{N}{2^{r-1}}$ is an integer. For instance, we can find an example see Example 7 for $r=3$, $N=12$ but we do not know the answer of the following question:

Question 2. Is there any example for $r=3$ and $N=10$ ? More precisely, can we say that $\mathbf{M P}(3,10)$ is nonempty?

Note that the following example can not be obtained by the constructions we mentioned above. 
Example 7. For $r=3, N=12, d=(0,0,-1, \ldots,-1)$ and $\mathcal{R}+\mathcal{C}=3$, consider

$$
\left[\begin{array}{rrrrrrrrrrrr}
0 & x & y^{2} & y z & z^{2} & 0 & 0 & 0 & 0 & 0 & 0 & 0 \\
0 & 0 & 0 & 0 & 0 & y^{2} & y z & 0 & z^{2} & 0 & 0 & 0 \\
0 & 0 & 0 & 0 & 0 & -x & 0 & -z & 0 & 0 & 0 & 0 \\
0 & 0 & 0 & 0 & 0 & 0 & -x & y & 0 & z & 0 & 0 \\
0 & 0 & 0 & 0 & 0 & 0 & 0 & 0 & -x & -y & 0 & 0 \\
0 & 0 & 0 & 0 & 0 & 0 & 0 & 0 & 0 & 0 & -z & 0 \\
0 & 0 & 0 & 0 & 0 & 0 & 0 & 0 & 0 & 0 & y & -z \\
0 & 0 & 0 & 0 & 0 & 0 & 0 & 0 & 0 & 0 & x & 0 \\
0 & 0 & 0 & 0 & 0 & 0 & 0 & 0 & 0 & 0 & 0 & y \\
0 & 0 & 0 & 0 & 0 & 0 & 0 & 0 & 0 & 0 & 0 & -x \\
0 & 0 & 0 & 0 & 0 & 0 & 0 & 0 & 0 & 0 & 0 & 0 \\
0 & 0 & 0 & 0 & 0 & 0 & 0 & 0 & 0 & 0 & 0 & 0
\end{array}\right]
$$

Note that we can obtain an example for $r=3$ and $N=4 s$ for every $s \geq 2$ by using the examples for $r=3, N=8$ and the example for $r=3, N=12$ and applying the chessboard construction as many times as necessary. If the answer to question 2 is negative, then one can ask the following question:

Question 3. Do there exist any periodicity results about nonemptiness of $\mathbf{M P}(r, N)$ ?

Another observation we make about these examples is that there always exists a sequence of permutations $\sigma_{1}<\sigma_{2}<\cdots<\sigma_{r}$ such that the image of the morphism contains a point from each Borel orbit corresponding to the these $\sigma_{i}$ 's and each pair of consecutive $\sigma_{i}$ 's consist of distinct transpositions. For example, putting $x=1$ and $y=0$ to $\psi$ in Example 2, we get a point in the Borel orbit corresponding to permutation $\sigma_{2}=(1,2)(3,4)$, and putting $x=0$ and $y=1$, we get $\sigma_{1}=(1,3)(2,4)$. Hence one could ask the following question:

Question 4. Given $\sigma$ in $\operatorname{MP}(r, N)$ does there always exists a morphism $\psi: \mathbb{P}_{k}^{r-1} \rightarrow V(d)-L(d)$ with a sequence permutations $\sigma_{1}<\sigma_{2}<\cdots<\sigma_{r}$ and points $X_{1}, X_{2}, \ldots, X_{r}$ in the image of $\psi$ such that $\sigma_{\psi}=\sigma$ and $X_{i}$ is in the Borel orbit of $\sigma_{i}$ for all $i$ and $\sigma_{i}$ and $\sigma_{i+1}$ has no common transpositions?

If the answer is affirmative to this question then one can say that the inequalities

$$
\frac{n(n+1)}{2} \leq \operatorname{dim}\left(B_{\sigma_{i}}\right) \leq n^{2}
$$

and

$$
\operatorname{dim}\left(B_{\sigma_{i}}\right)+\left\lceil\frac{n}{2}\right\rceil \leq \operatorname{dim}\left(B_{\sigma_{i+1}}\right)
$$

hold and they give the inequality $N \geq 2 r$.

Note that Allday and Puppe [3] have related results: If $k, A, r, N$, and $M$ are as in Conjecture 1, then they prove $N \geq 2 r$. Moreover, Avramov, Buchweitz and Iyengar [4] verified that $N \geq 2 r$ in a more general case.

\section{References}

[1] A. Adem, W. Browder, The free rank of symmetry of $\left(S^{n}\right)^{k}$, Invent. Math. 92 (2) (1988) 431-440, https://doi.org/10. 1007/BF01404462. 
[2] C. Allday, V. Puppe, Bounds on the torus rank, in: Transformation Groups, Poznań 1985, in: Lect. Notes Math., vol. 1217, Springer, Berlin, 1986, pp. 1-10.

[3] C. Allday, V. Puppe, Cohomological Methods in Transformation Groups, Camb. Stud. Adv. Math., vol. 32, Cambridge University Press, Cambridge, 1993.

[4] L.L. Avramov, R.-O. Buchweitz, S. Iyengar, Class and rank of differential modules, Invent. Math. 169 (1) (2007) 1-35, https://doi.org/10.1007/s00222-007-0041-6.

[5] D.J. Benson, J.F. Carlson, Complexity and multiple complexes, Math. Z. 195 (2) (1987) 221-238, https://doi.org/10.1007/ BF01166459.

[6] W. Browder, Cohomology and group actions, Invent. Math. 71 (3) (1983) 599-607, https://doi.org/10.1007/BF02095996.

[7] G. Carlsson, On the rank of abelian groups acting freely on $\left(S^{n}\right)^{k}$, Invent. Math. 69 (3) (1982) 393-400, https://doi.org/ 10.1007/BF01389361.

[8] G. Carlsson, On the homology of finite free $(\mathbf{Z} / 2)^{n}$-complexes, Invent. Math. 74 (1) (1983) 139-147, https://doi.org/10. 1007/BF01388534.

[9] G. Carlsson, Free $(\mathbf{Z} / 2)^{k}$-actions and a problem in commutative algebra, in: Transformation Groups, Poznań 1985, in: Lect. Notes Math., vol. 1217, Springer, Berlin, 1986, pp. 79-83.

[10] G. Carlsson, Free $(\mathbf{Z} / 2)^{3}$-actions on finite complexes, in: Algebraic Topology and Algebraic K-Theory, Princeton, N.J., 1983, in: Ann. Math. Stud., vol. 113, Princeton Univ. Press, Princeton, NJ, 1987, pp. 332-344, https://press.princeton. edu/titles/2548.html.

[11] P.E. Conner, On the action of a finite group on $S^{n} \times S^{n}$, Ann. Math. (2) 66 (1957) 586-588, https://doi.org/10.2307/ 1969910.

[12] M.P. Drazin, J.W. Dungey, K.W. Gruenberg, Some theorems on commutative matrices, J. Lond. Math. Soc. 26 (1951) 221-228, https://doi.org/10.1112/jlms/s1-26.3.221.

[13] A. Heller, A note on spaces with operators, Ill. J. Math. 3 (1959) 98-100, http://projecteuclid.org/euclid.ijm/1255455001.

[14] K. Hoffman, R. Kunze, Linear Algebra, second edition, Prentice-Hall, Inc., Englewood Cliffs, N.J., 1971.

[15] S.B. Iyengar, M.E. Walker, Examples of finite free complexes of small rank and homology, Acta Math. (2018), in press, https://arxiv.org/abs/1706.02156.

[16] D. Karagueuzian, B. Oliver, J. Ventura, The components of a variety of matrices with square zero and submaximal rank, in: Commutative Algebra, in: Lect. Notes Pure Appl. Math., vol. 244, Chapman \& Hall/CRC, Boca Raton, FL, 2006, pp. $151-164$.

[17] N.H. McCoy, On the characteristic roots of matric polynomials, Bull. Am. Math. Soc. 42 (8) (1936) 592-600, https:// doi.org/10.1090/S0002-9904-1936-06372-X.

[18] A. Melnikov, $B$-orbits in solutions to the equation $X^{2}=0$ in triangular matrices, J. Algebra 223 (1) (2000) 101-108, https://doi.org/10.1006/jabr.1999.8056.

[19] O.B. Okutan, E. Yalçın, Free actions on products of spheres at high dimensions, Algebraic Geom. Topol. 13 (4) (2013) 2087-2099, https://doi.org/10.2140/agt.2013.13.2087.

[20] M. Refai, Group actions on finite CW-complexes, Indian J. Pure Appl. Math. 24 (4) (1993) 245-255, http://insa.nic.in/ writereaddata/UpLoadedFiles/IJPAM/20005a57_245.pdf.

[21] B.D. Rothbach, Borel Orbits of X2 = 0 in gl(n), ProQuest LLC, Ann Arbor, MI, thesis, (Ph.D.)-University of California, Berkeley, 2009, http://gradworks.umi.com/33/83/3383430.html.

[22] H. Shapiro, Simultaneous block triangularization and block diagonalization of sets of matrices, Linear Algebra Appl. 25 (1979) 129-137, https://doi.org/10.1016/0024-3795(79)90012-0.

[23] P.A. Smith, Permutable periodic transformations, Proc. Natl. Acad. Sci. USA 30 (1944) 105-108, http://www.jstor.org/ stable/87918.

[24] M.E. Walker, Total Betti numbers of modules of finite projective dimension, version 1 of https://arxiv.org/abs/1702.02560, 2017.

[25] E. Yalçın, Group actions and group extensions, Trans. Am. Math. Soc. 352 (6) (2000) 2689-2700, https://doi.org/10.1090/ S0002-9947-00-02485-5. 